\title{
Cold agglutinin disease associated with mycoplasma infection in an individual with type 2 diabetes: An atypical case
}

\author{
Chandrama Shrestha, Min Liu, Zhaohui Mo ${ }^{\#}$ \\ The Endocrinology Department of the Third Xiangya Hospital, Central South University, Changsha, China; \\ \#Corresponding Author: easd04mzh@126.com
}

Received 18 July 2012; revised 20 August 2012; accepted 19 September 2012

\begin{abstract}
Cold Agglutinin Disease is a hemolytic anemia associated with cold reactive autoantibodies. Although the acute form of cold agglutinin disease can be attributed to autoimmune or infectious diseases and lymphoproliferative diseases, it has, to the best of our knowledge, so far, never been reported as secondary to Mycoplasma pneumonia in a type 2 diabetic individual. In this paper, we report a case of cold agglutinin disease following Mycoplasma pneumonia in a 47year-old female patient with type 2 diabetes and schizophrenia. Cold agglutinin test and direct Coombs' test was positive. Anti-mycoplasma antibody titre by complement fixation was high (AntiIgG was negative and Anti-C3d was positive) and was accompanied by hemolytic anemia. Her general condition, including Cold Agglutinin Disease improved after conservative therapy with antibiotics, hypoglycemic agents and short-term use of steroids. The patient recuperated and was discharged in good health after 7 days' stay in the hospital. She remains clinically well with no recurrence of anemia. The simultaneous occurrence of cold agglutinin disease, mycoplasma infection and diabetes mellitus is rare and accumulation of case reports is required to gain better insight of this case scenario.
\end{abstract}

Keywords: Cold Agglutinin Disease; Diabetes Mellitus; Mycoplasma

\section{BACKGROUND}

Although more than a century has elapsed since cold agglutinins were discovered, a case of cold agglutinin disease complicated by mycoplasma infection in a type 2

\footnotetext{
"The authors declare that they have no competing interests.
}

diabetic individual has, in our knowledge, so far, never been reported in medical literature.

After Landsteiner first described cold agglutinins in the early twentieth century, their pathological action against red blood cells (hemolytic anemia) and blood vessels (Raynaud's syndrome) was elaborated by Clough and Iwai. Half a century has elapsed since Scubothe coined the term Cold Agglutinin Disease. Cold Agglutinin Disease is characterized by an auto-antibody which has the capability to agglutinate red blood cells at temperatures lower than that of the body and subsequently to induce complement activation, as a consequence of which there is lysis of red blood cells, resulting in impaired blood flow to the digits, nose and ears. Cold agglutinins bind to erythrocyte surface antigens at a temperature optimum of 0 - 4 degrees centigrade [1,2]. In contrast to polyclonal cold agglutinin in healthy individuals, monoclonal cold agglutinin often have highthermal amplitude, which plays an important role owing to their pathogenicity at around 37 degree centigrade [1-4]. The auto-antibody involved is almost invariably an IgM, less frequently an IgA or IgG and complement activation generally occurs between 20 and 25 degrees centigrade.

Typical clinical manifestation of cold agglutinin disease includes cold-induced circulatory symptoms as well as hemolytic anemia of varying degrees of severity and episodes of hemoglobinuria and acrocyanosis, which tend to exacerbate or alleviate upon exposure to low temperatures. Cold agglutinin antibodies are mainly specific for the $\mathrm{I} / \mathrm{i}$ and $\mathrm{H}$ red blood cells membrane systems [5] and the possible triggering factors include various infectious agents (e.g. mycoplasma, Ebstein Barr virus) and lymphoproliferative disorders (Waldenstroms macrogammaglobulinemia).

\section{ETHICAL CONSIDERATION}

Written informed consent was obtained from the patient for the publication of this case report. All the tests 
were performed with the approval of Third Xiangya Hospital Ethics committee and conformed to the Helsinki Declaration.

\section{CASE PRESENTATION}

A 47-year-old lady, a known case of diabetic nephropathy and schizophrenia, presented to the out-patient department of our hospital, with complaints of cough associated with the production of foamy white sputum and occasional blurred vision and dizziness, all of which started approximately ten days after being discharged on anti-inflammatory drugs, antibiotics, and insulin 70/30 on a bd regime: $(16+8) \mathrm{IU}$, from a hospital-stay of 6 days for DKA, lower lobe infection of left lung and fatty liver. There was no family history of hemolytic attacks. On general physical examination, the patient appeared ill and pale. Puffy face, purplish discoloration of the fingers (Raynaud's phenomenon) and edematous lower limbs were evident on further examination. Blood pressure, pulse rate and temperature were within normal limits. Bilateral crepitations could be heard on chest auscultation. Other findings on physical examination was unremarkable. Her random plasma glucose level was found to be $18.0 \mathrm{mmol} / \mathrm{l}$ and was therefore transferred to the inpatient department for further evaluation and management. Macroscopically visible auto-agglutination could be noted in the tube containing the anti coagulated sample within one minute. Prudent observation of the tube revealed that the auto-agglutination disappeared at 37 degree centigrade. Direct Coombs' Test was positive and the anti-mycoplasma antibody titre by complement fixation was high.(Anti-IgG was negative and Anti-C3d was positive). The peripheral blood examination confirmed agglutination. Further, anisopoikilocytosis and polychromasia could be seen on peripheral smear. Subsequent blood tests revealed leukocytosis $\left(14.39 \times 10^{9} / \mathrm{L}\right)$, anemia $(54 \mathrm{~g} / \mathrm{L})$ and hyperbilirubinemia (total bilirubin = $7.9 \mathrm{umol} / \mathrm{L}$ ). Chest X-ray showed diffuse small nodular infiltrates over both lung fields. Abdominal ultrasonography revealed severe fatty liver, mild splenomegaly (thickness $=44 \mathrm{~mm}$ ) and solitary kidney (right kidney absent). A bone marrow aspiration was performed and then sent for biopsy to eliminate the possibility of an underlying leukemia. Based on the characteristics discussed in the preceding paragraphs and available literature, a diagnosis of cold agglutinin disease complicated by mycoplasma infection and diabetes mellitus was made. The patient was kept warm. Cold agglutinin therapy was pursued, along with systemic antibiotics, hypoglycemic agents and a short-term use of steroids (intravenous administration of dexamethasone $5 \mathrm{mg}$ and $10 \mathrm{mg}$ each on two consecutive days respectively), after which the patient recuperated and this phenomenon didn't reappear.
Fever subsided, hemoglobin level reached $74 \mathrm{~g} / \mathrm{L}$ and the leukocyte count declined to normal values. Transfusion therapy was not required. The patient improved and was discharged in good health after seven days. She was thoroughly counseled regarding her health status and precaution measures were discussed (i.e. keeping herself warm).

\section{DISCUSSION}

There has been few reported cases of cold agglutinin disease secondary to mycoplasma infection but we believe this to be the first report of its association with Mycoplasma pneumonia in conjunction with diabetes mellitus. Though Mycoplasma pneumonia infections are often asymptomatic, multiple organ systems can be affected. Respiratory tract involvement and extra-pulmonary complications in cold agglutinin disease manifest in $3 \%-10 \%[6,7]$ and $25 \%$ of the patients respectively and autoimmune reactions supposedly play a role in their pathogenesis [8]. Antibody mediated hemolysis is supposedly caused by the formation of cold agglutinins in $10 \%$ patients with Mycoplasma pneumonia infections [9].

Cold agglutinins are IgM auto-antibodies which effectively activate the classical complement cascade. In cold hemagglutinin disease, complement is the only protein detected in the red cells in the majority of cases, since IgM dissociates from red cells at higher temperatures. Regulatory proteins convert the red cell bound C3 and C4, to C3d, C3dg and C4D [10]. It is the anti-Cd3 component of polyspecific AHG (anti-C3d and anti-IgG) that is responsible for the positive Direct Coomb's test. In our patient, hematologic and biochemical evidence pointed towards the diagnosis of cold agglutinin disease.

In our case study, there was evidence of recently acquired Mycoplasma pneumonia infection (positive IgM for M. pneumoniae). Our patient had cold agglutinin titre of $1: 80$. The reactivity was inversely proportional to temperature and completely disappeared at 37 degree centigrade. In a case reported by Wilson et al. [11], the titre of auto-antibodies was 1:512 and the patient also had recurrent arterial thrombosis, reflecting an autoimmune phenomenon with damage to the endothelium. In another case reported by Basu et al. [12], the patient had a cold agglutinin titre of 1:256 and the auto-antibodies were most reactive at 4 degree centigrade. The reactivity decreased at warmer temperatures (22 - 30) degree centigrade and completely disappeared at 37 degree centigrade, emphasizing the fact that it is the reactivity of the cold agglutinin at warm temperatures and not the antibody titre that correlates with the severity of the disease.

According to available literature, the anemia in cold agglutinin disease is variable and severe hemolytic anemia is rare [13] and is usually associated with marked 
pulmonary involvement [14]. A racial predilection has not been reported for cold agglutinin disease, neither noted among sex differences although some researchers report a female predilection in older populations. Autoimmune hemolytic anemia appears to be more common in male children and female adolescents. The data available is insufficient regarding the possible geographic variation on the prevalence of cold agglutinin disease.

Keeping the patient warm and management of associated diseases remain the mainstay of treatment. Strict avoidance to cold should be emphasized. When cold agglutinin disease is secondary, as in the case of infectious disease or lymphoma, concurrent with instituting a cold avoidance measure, the key components of treatment include identification and management of the underlying infection and/or malignancy, whichever the case. Most patients of cold agglutinin disease secondary to $M y$ coplasma pneumonia infection recover with supportive care $[15,16]$. It's possible that the role of antibiotics cannot be overestimated in mycoplasma infection associated with hemolytic anemia [13], however treatment of the underlying mycoplasma infection has been associated with more rapid resolution of the hemolytic process [15]. Furthermore, the use of packed red blood cell transfusions should be limited on grounds of the fact that following this, hemolysis would ensue in patients with autoimmune hemolytic anemia [17]. Using intravenous immunoglobulin in patients with cold agglutinin disease superseded by Mycoplasma pneumonia infection has been proved beneficial in inhibiting hemolysis until spontaneous clearance of the IgM antibodies occurs [18, 19].

Rampant use of corticosteroids, azathioprine, interferon and purine nucleoside analogues for treating primary cold agglutinin disease has been sparsely recommended [20]. Corticosteriods, plasmapheresis and cytotoxic drugs have not shown much promise in the treatment of secondary cold agglutinin disease. However, in a case reported by Nasu [21], it is stated that corticosteroid therapy is sometimes necessary for the treatment of marked anemia. This was replicated in a case of cold agglutinin disease associated with Mycoplasma pneumonia, reported by Inoue F. et al. stating that obvious clinical improvement was noted after treating with minocycline and prednisolone [20]. Our case of cold agglutinin disease complicated by mycoplasma and diabetes improved with a short course of dexamethasone.

\section{CONCLUSION}

Co-occurrence of cold agglutinin disease, mycoplasma infection and diabetes mellitus is rare and accumulation of case reports is imperative to enhance our knowledge of this condition. This case was encountered incidentally in the inpatient department and then further analyzed in accordance with the patient's clinical profile. The importance of reporting this case is to point out the possibility of multiple organ involvement in Mycoplasma pneumonia infections that might inadvertently be overlooked.

\section{REFERENCES}

[1] Ulvestad, E., Berentsen, S., Bo, K. and Shammas, F.V. (1999) Clinical immunology of chronic cold agglutinin disease. European Journal of Haematology, 63, 259-266. doi:10.1111/j.1600-0609.1999.tb01887.x

[2] Olesen, H. (1966) Thermodynamics of the cold agglutinin reaction. Scandinavian Journal of Clinical \& Laboratory Investigation, 18, 1-15. doi:10.3109/00365516609065601

[3] Rosse, W.F. and Adams, J.P. (1980) The variability of hemolysis in the cold agglutinin syndrome. Blood, 56, 409-416

[4] Zilow, G., Kirschfink, M. and Roelcke, D. (1994) Red cell destruction in cold agglutinin disease. Transfusion Medicine and Hemotherapy, 21, 410-415. doi:10.1159/000223021

[5] Geoff, D. (1995) Human blood groups. Blackwell Science Ltd., London, 53-61.

[6] Clyde, W.A., Jr. (1993) Clinical overview of typical Mycoplasma pneumonia infections. Clinical Infectious Diseases, 17, S32-S36.

[7] Foy, H.M. (1993) Infections caused by Mycoplasma pnemoniae and possible carrier state in different populations of patients. Clinical Infectious Diseases, 17, S37S46. doi:10.1093/clinids/17.Supplement_1.S37

[8] Waites, K.B. and Talkington, D.F. (2004) Mycoplasma pneumonia and its role as a human pathogen. Clinical Microbiology Reviews, 17, 697-728. doi:10.1128/CMR.17.4.697-728.2004

[9] Salama, A. (2004) Acquired immune hemolytic anemias. Therapeutische Umschau, 61, 178-186. doi:10.1024/0040-5930.61.2.178

[10] Silberstein, L.E. and Cunningham, M.J. (2007) Autoimmune hemolytic anemias. In: Blood Banking and Transfusion Medicine: Basic Principles and Practice, 2nd Edition, Elsevier, Churchill Livingstone, Section III, Chapter 41, 557-570. doi:10.1016/B978-0-443-06981-9.50046-6

[11] Wilson, M.L., Menjivar, E., Kalapatapu, V., Hand, A.P., Garber, J. and Ruiz, M.A. (2007) Mycoplasma pneumoniae associated with hemolytic anemia, cold agglutinins and recurrent arterial thrombosis. Southern Medical Journal, 100, 215-217. doi:10.1097/01.smj.0000254212.35432.99

[12] Basu, S., Saifudeen, A. and Kaur, P. (2009) Transient cold agglutinin disease with mycoplasma infection. Journal of the Association of Physicians of India, 57, 653-654.

[13] Daxbock, F., Zedtwitz-Hiebenstein, K., Burgmann, H. and Graninger, W. (2001) Severe hemolytic anemia and excessive leukocytosis masking Mycoplasma pneumonia. Annals of Hematology, 80, 180-182. doi:10.1007/s002770000250

[14] Cherry, J.D. and Ching, N. (2004) Mycoplasma and urea- 
plasma infections. Textbook of Pediatric Infectious Diseases, Vol. 2, 3rd Edition, Saunders, Philadelphia, 25162531.

[15] Gertz, M.A. (2006) Cold hemolytic syndrome. American Society Hematology Education Program Book, 2006, 1923. doi:10.1182/asheducation-2006.1.19

[16] Gehrs, B.C. and Friedberg, R.C. (2002) Autoimmune hemolytic anemia. American Journal of Hematology, 69, 258-271. doi:10.1002/ajh.10062

[17] Logue, G.L., Rosse, W.F. and Gockerman, J.P. (1973) Measurement of the third component of complement bound to red blood cells in patients with cold agglutinin syndrome. Journal of Clinical Investigation, 52, 493-501. $\underline{\text { doi:10.1172/JCI107206 }}$
[18] Hoppe, B., Gaedicke, G., Kiesewetter, H. and Salama, A.R. (2004) Response to intravenous immunoglobulin $G$ in an infant with immunoglobulin A-associated autoimmune haemolytic anemia. Vox Sang, 86, 151-153. doi:10.1111/j.0042-9007.2004.00392.x

[19] Wang, J.L., Ho, M.Y. and Shen, E.Y. (2004) Mycoplasma pneumonia infection associated with hemolytic anemia report of one case. Acta Pediatr Taiwan, 45, 293-295.

[20] Inoue, F., Miyake, N., Yamasowa, N., Ohno, T., Takamatsu, T., Okada, T., Mizumoto, et al. (1992) Cold agglutinin hemolytic anemia complicating Mycoplasma pneumonia. Rinsho Ketsueki, 33, 801-805.

[21] Nasu, K. (1996) Mycoplasma infection and hemolytic anemia. Nippon Rinsho, 54, 2545-2549. 